\title{
Relativity on Rotated Graph Paper
}

\author{
Roberto B. Salgado \\ Department of Physics and Astronomy, \\ Bowdoin College, Brunswick, Maine 04011 USA*
}

\begin{abstract}
We present visual calculations in special relativity using spacetime diagrams drawn on graph paper that has been rotated by 45 degrees. The rotated lines represent lightlike directions in Minkowski spacetime, and the boxes in the grid (called "light-clock diamonds") represent units of measurement modeled on the ticks of an inertial observer's lightclock. We show that many quantitative results can be read off a spacetime diagram by counting boxes, using a minimal amount of algebra. We use the Doppler Effect, in the spirit of the Bondi $k$-calculus, to motivate the method.
\end{abstract}




\section{INTRODUCTION}

Soon after Einstein's 1905 paper ${ }^{\underline{1}}$ on special relativity revolutionized our understanding of space and time, Minkowski ${ }^{2}$ introduced the "spacetime diagram" (akin to an ordinary position vs. time graph) on which relativistic concepts can be encoded geometrically. Unfortunately, due to the spacetime diagram's underlying non-euclidean geometry, it may not be evident where to place tickmarks representing units of elapsed-time and spatial-distance for various inertial observers in special relativity. Without such tickmarks, spacetime diagrams may be difficult to draw, to use for calculations, and to interpret physically. Although one could draw the invariant hyperbolas or perform algebraic calculations with the Lorentz Transformations, either approach may be too sophisticated for a novice.

To help overcome these difficulties, we present a graphical method of calculation using a $(1+1)$-dimensional Minkowski spacetime diagram that is drawn on ordinary graph paper rotated by 45 degrees. The rotated grid will be interpreted (in Sec. VG) as the radartime light-cone coordinates ${ }^{3}$ of an inertial observer, where the unit squares (later called "light-clock diamonds") mark off tickmarks for that observer's coordinate system.

Using a geometric construction, we determine an analogous grid of unit rectangles to mark off tickmarks for another inertial observer's coordinate system. When the relativevelocities between inertial observers are rational numbers, calculations in special relativity can be read off the rotated grid by counting unit rectangles and doing simple arithmetic (without the explicit use of relativistic formulas). This is the main result of this article.

The construction (to be described in detail in Sec. IV]) exploits the following geometrical facts ${ }^{4} \underline{\underline{7}}$ which will be motivated and interpreted physically: (1) a rectangle in the grid [with its lightlike sides] (later called a "causal diamond" $\underline{\underline{8}}$ ) has an area ${ }^{9}$ proportional to the squareinterval ${ }^{10}$ of its timelike-diagonal, and (2) a Lorentz boost transforms a rectangle in the grid into another rectangle in the grid with equal area but different aspect ratio, stretched in one lightlike direction by the Doppler factor $k$ and compressed in the other lightlike direction by the same factor. These geometrical facts encode properties of the invariant hyperbolas of Minkowski spacetime.

We begin in Sec. II with an explanation of Alice's Light Clock and its representation on the rotated graph paper. In Sec. III, we introduce the causal and light-clock diamond and some additional terminology useful for describing and reasoning with spacetime diagrams. 
We construct Bob's Light Clock in Sec. IV] in two different ways, using the Doppler Effect. (Although our methods are in the spirit of the Bondi $k$-calculus, $\frac{11}{1}$ no familiarity with it is assumed.) We apply our methods to some standard examples from special relativity in Sec.V. In particular, in subsection $\mathrm{VG}$, we develop a presentation based on radar-methods, which provides operational definitions of physical quantities. We conclude in Sec. VI with an algebraic interpretation of the construction.

With our graphical method, we are able to place emphasis on the physical interpretation first, which may be followed by the development of the relativistic formulas (to handle more general situations), if desired. The simplicity of the method suggests that it could be useful in an introductory course. (The author has used aspects of the method in such courses.)

\section{THE TICKS OF ALICE'S LIGHT-CLOCK}

We begin the construction by motivating the interpretation of the unit squares in the rotated grid. Consider an inertial observer, Alice, at rest in her reference frame, carrying a mirror a constant distance $D$ away. Alice emits a light flash (traveling with speed $c$ ) which reflects off the distant mirror and returns (at speed $c$ ) to her after a round-trip elapsed time $2 D / c$. If this returning light-signal is immediately reflected back, this functions like a clock, called the light-clock. $1, \underline{12}-\underline{14}$ Since we wish to regard time as a more primitive concept than space, let us declare that round-trip time to be 1 "tick", so that $D=(1 / 2) c$ tick $=$ (1/2) "light-tick" [analogous to the light-year as a unit of distance]. For convenience, let us define $d=(D / c)$ so that the spatial displacement $d$ is also measured in "ticks." Thus, $d$ measures the duration for light to travel the desired spatial displacement. In these units, $c=(1$ tick $) /$ tick, or simply $c=1$. More generally, velocities will be dimensionless and will be denoted by the symbol $\beta$.

For symmetry, consider Alice with two such mirrors, one to the right (the direction in which Alice faces) and the other to the left, so that they are spatially separated by 1 tick. Using the rotated grid, the worldlines of Alice and her mirrors (drawn as dotted lines) and the associated light-signals are shown on the spacetime diagram in Fig. 1, The parallelogram $O M T N$ will be used to represent one tick of Alice's longitudinal light-clock. Assuming translation symmetry in spacetime, Alice can set up a coordinate system in which the squares in the grid are modeled after this parallelogram. 


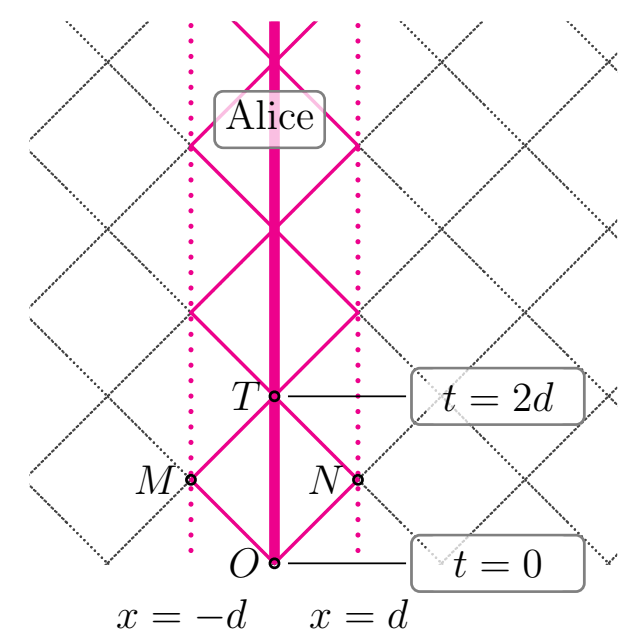

FIG. 1: Alice's Longitudinal Light-Clock in her frame of reference. Alice emits light-signals at $O$ which reflect off her two mirrors (at $x=-d$ and $x=d$ ) to be received by Alice after an elapsed-time $\Delta t=2 d$. The resulting parallelogram $O M T N$ defines 1 "tick" of Alice's clock (so, $d=(1 / 2)$ tick), which can be used to set up a coordinate system for Alice.

\section{CAUSAL DIAMONDS}

\section{A. Visualizing Proper-time with Causal Diamonds}

We pause to introduce some terminology which will be useful for interpreting the diagrams

physically and operationally, $, \underline{11}, 19,20$ in the spacetime viewpoint. This brief section assumes some familiarity with the spacetime geometry of special relativity ${ }^{2,11,15}=22$ and is intended to give the educator an overview of the construction. Aspects of this section were presented to students after developing the construction in Sec. IVB and then treating some examples.

The future-light-cone of an event $O$ is the set of events met by light-signals emitted at $O$. Similarly, the past-light-cone of $O$ is the set of events whose emitted light-signals meet $O$. In Fig. 1, the future-light-cone of $O$ is the pair of rays from $O$ along the grid through $O M$ and $O N$, and the past-light-cone of event $T$ is the pair of rays from $T$ along the grid through $T M$ and $T N$.

Consider now the parallelogram $O M T N$ in Fig. 1, whose sides are traced out by the light-signals of the light-clock. Note that diagonal OT (along Alice's worldline) is futuretimelike, and diagonal $M N$ is spacelike. In fact, the reflection events $M$ and $N$ are said to be "simultaneous according to Alice" since light-signals from event $O$ to events $M$ and $N$ are received at a common event $T$. (Geometrically, events on the intersection of the future-light cone of $O$ and the past-light-cone of $T$ are simultaneous according to Alice.) 
Moreover, Alice regards events $M$ and $N$ as mutually simultaneous with her "half-tick," the midpoint event of $O T$. Thus, physically, $M N$ represents a purely-spatial direction for Alice when her clock reads $t=\frac{1}{2}$ tick. This operationally defines the geometric notion that the spacelike-diagonal $M N$ is "spacetime-perpendicular" 23 to the timelike-diagonal OT. In fact, Alice's spatial $x$-axis (when her clock reads $t=0$ ticks) is the ray from event $O$ that is parallel to $M N$. See Fig. 2, (In the reference frame shown in Figs. 1 and 2, $M N$ happens to also be perpendicular to $O T$ in the usual Euclidean sense.)

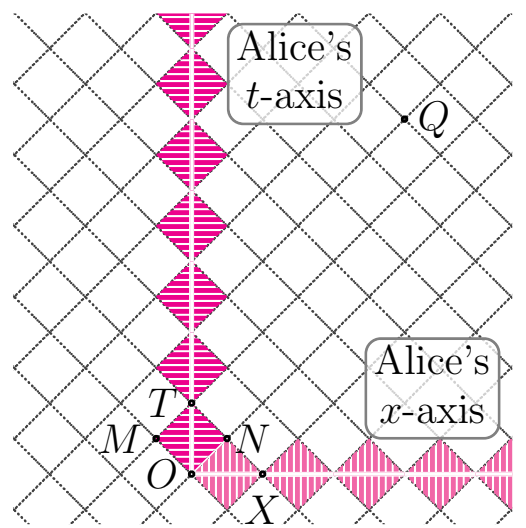

FIG. 2: Alice's Rectangular Coordinate System uses the diagonals of her light-clock diamonds to locate events. Alice's time-axis is marked by a string of light-clock diamonds with their timelikediagonals along $\overrightarrow{O T}$ on her worldline. Alice's spatial $x$-axis is marked by a string of light-clock diamonds with their spacelike-diagonals along $\overrightarrow{O X}$, on a line of simultaneity according to Alice (parallel to $M N$ ) through event $O$. For example, since $\overrightarrow{O Q}=5 \overrightarrow{O T}+3 \overrightarrow{O X}$, Alice assigns event $Q$ rectangular coordinates $\left(t_{Q}^{\text {(Alice) }}, x_{Q}^{(\text {Alice })}\right)=(5,3)$.

Note, however, that the lightlike sides $O M$ and $O N$ are not spacetime-perpendicular $\underline{24}$ to each other. Thus, $O M T N$ is neither a square nor a rectangle in Minkowskian geometry. Instead, we will refer to parallelogram $O M T N$ as the "causal diamond of $O T$ " interior is the intersection of the future of event $O$ and the past of event $T$ ). In what follows, we will develop the idea that causal diamonds are quantitative visualizations of the elapsed proper time along its timelike diagonal.

\section{B. Coordinates Systems for Counting Light-Clock Diamonds}

When the causal diamond of $O T$ (that is, parallelogram $O M T N$ ) is chosen as a standard to mark one tick of Alice's light-clock, we will refer to it as a "light-clock diamond of Alice," or simply as "Alice's diamond." This provides a standard unit with which Alice can construct a coordinate system. Since our emphasis is on counting diamonds, we have 
adopted the following choice of coordinates. Fig. 2 shows Alice's rectangular coordinate system: $(t, x)$, where $t$ and $x$ are dimensionless numbers that count the number of timelike and spacelike diagonals of Alice's light-clock diamond. Fig. 3 shows Alice's "light-cone coordinate" system: $:^{-3}(u, v)$, where $u$ and $v$ are dimensionless numbers that count the number of right- and left-pointing lightlike edges of Alice's diamond. This coordinate system will be physically interpreted in Sec. VG.

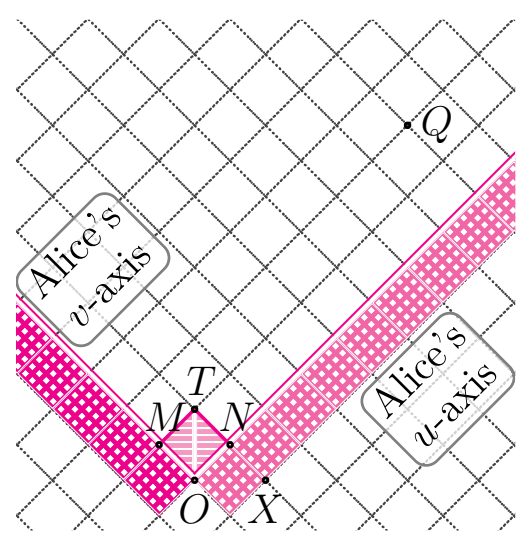

FIG. 3: Alice's Light-Cone Coordinate System uses the edges of her light-clock diamonds to locate events. Alice's $u$ - and $v$-axes are marked by strings of light-clock diamonds with their lightlikeedges along $\overrightarrow{O N}$ and $\overrightarrow{O M}$, respectively, on the light-cone of event $O$. Since $\overrightarrow{O T}=\overrightarrow{O N}+\overrightarrow{O M}$ and $\overrightarrow{O X}=\overrightarrow{O N}-\overrightarrow{O M}$, we have $\overrightarrow{O Q}=8 \overrightarrow{O N}+2 \overrightarrow{O M}$. So, Alice assigns event $Q$ the coordinates $\left(u_{Q}^{(\text {Alice })}, v_{Q}^{(\text {Alice })}\right)=(8,2)$. In Sec. $\mathrm{VG}$, we will show that the values of $u$ and $v$ have direct physical interpretation as clock-readings in Alice's radar experiment to locate event $Q$.

Using these coordinate systems, an arbitrary vector $\overrightarrow{O Q}$ can be expressed as

$$
\overrightarrow{O Q}=t_{Q} \overrightarrow{O T}+x_{Q} \overrightarrow{O X}=u_{Q} \overrightarrow{O N}+v_{Q} \overrightarrow{O M}
$$

Since

$$
\overrightarrow{O T}=\overrightarrow{O N}+\overrightarrow{O M} \quad \text { and } \quad \overrightarrow{O X}=\overrightarrow{O N}-\overrightarrow{O M}
$$

we find that the coordinate systems are related by

$$
u \equiv t+x \quad \text { and } \quad v \equiv t-x \text {. }
$$

Note that the product $u v$ is equal to the square-interval

$$
u v=t^{2}-x^{2},
$$


which plays an important role in special relativity. These relationships can be verified for the events shown in Figs. 2 and 3. Further aspects of our choice of coordinates systems will be discussed in Sec. VI.

\section{BUILDING BOB'S LIGHT-CLOCK}

Now, consider another inertial observer, Bob, moving with velocity $\beta=(3 / 5)$ (for numerical convenience) according to Alice. Refer to Fig. 4. Let $O V$ refer to Bob's worldline and let event $F$ on $O V$ mark off one tick from Bob's clock since event $O$. Given $\beta$, we use Alice's ticks to determine the line $O V$. The problem is to locate event $F$ on $O V$.

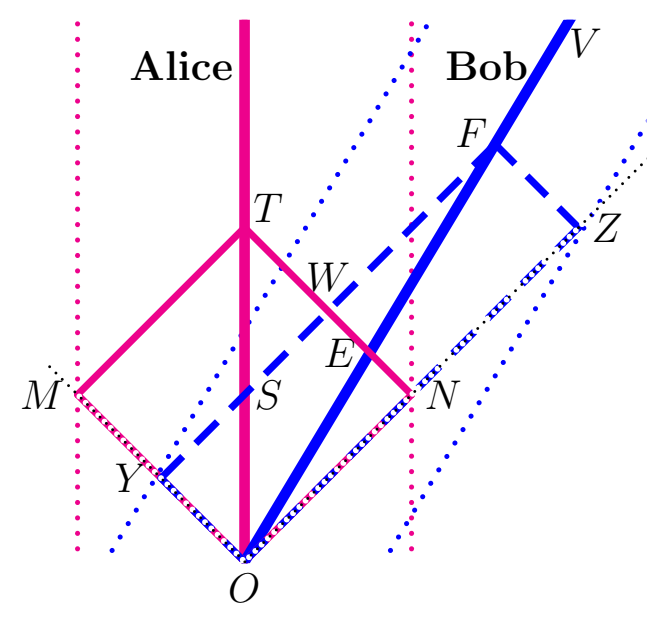

FIG. 4: Bob's Longitudinal Light-Clock in Alice's frame of reference. Bob, traveling with velocity $(3 / 5)$ according to Alice, emits light-signals at $O$ which reflect off his two mirrors to be received by Bob after an elapsed-time of 1 tick on his clock. The resulting parallelogram $O Y F Z$ with timelikediagonal $O F$ would define Bob's light-clock diamond, which can be used to set up a coordinate system for Bob. So, given parallelogram $O M T N$ and $O V$, we wish to determine the event $F$ on $O V$ such that $t_{T}^{(\text {Alice })}=t_{F}^{(B o b)}$.

In accordance with the Relativity Principle and the Speed of Light Principle, $\frac{1}{2}$ we expect that the first tick of Bob's identically-constructed longitudinal light-clock will be drawn as the causal diamond of $O F$. Starting from the causal diamond of OT (one tick of Alice's light-clock), we will now construct the causal diamond of $O F$ (one tick of Bob's light-clock). The key result we will deduce is that the areas of these diamonds are equal. 


\section{A. A geometrical proof}

We will use the Doppler Effect (in the spirit of the Bondi $k$-calculus ${ }^{11}$ ) to elaborate on a geometric argument from Mermin. ${ }^{5}$ Our goal is to highlight the role of physical laws in the construction. Since this argument may be too abstract for students, we will present another demonstration in Sec. B (which I have used with students). Some further aspects of the construction are treated algebraically in Sec. VI. (As mentioned earlier in the introduction, no familiarity with the Bondi $k$-calculus is assumed.)

Refer again to Fig. 4. Start with the causal diamond of $O T$ and Bob's worldline $O V$. Note the intersection events: the given event $O$ and the event $E$ on $T$ 's past-light-cone. Interpret these events $O$ and $E$ as Bob emitting two light-signals which are received by Alice at events $O$ and $T$, respectively. Along segment $O E$, let $t_{E}^{(B o b)}$ represent the period between emissions according to Bob's clock, and along segment $O T$, let $t_{T}^{(B o b)}$ represent the period between receptions according to Alice's clock. These periods (in triangle $O E T$ ) are related by

$$
t_{T}^{(\text {Alice })}=k_{B o b}^{(\text {Alice })} t_{E}^{(\text {Bob })}
$$

where $k_{B o b}^{(\text {Alice })}$ is a proportionality factor determined by Alice that depends on the relative speed $\beta$, but is independent of $t_{T}^{(\text {Alice) }}$. In passing, we mention that this factor, which we call the Bondi-Doppler $k$-factor, is equal to the familiar Doppler factor

$$
k=\sqrt{(1+\beta) /(1-\beta)},
$$

as we show in Sec. VG (Eq. 29). We do not need this explicit expression in this section.

With an arbitrary event $F$ along $O V$, construct the causal diamond of $O F$ and the corresponding intersection events $O$ and $S$ on Alice's worldline. Similarly, we have (in triangle $O S F$ )

$$
t_{F}^{(B o b)}=k_{\text {Alice }}^{(\text {Bob })} t_{S}^{(\text {Alice })},
$$

where $k_{\text {Alice }}^{(B o b)}$ is the analogous factor determined by Bob. By the Relativity Principle, since Alice and Bob are conducting identical experiments on each other, we must have

$$
k_{\text {Bob }}^{(\text {Alice })}=k_{\text {Alice }}^{(B o b)},
$$


which we will now call $k_{\text {Alice,Bob }}$, or simply $k$ when there is no ambiguity.

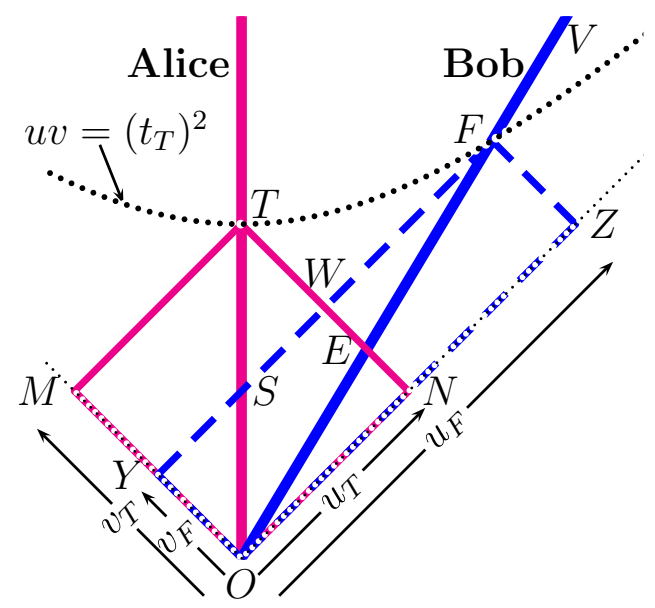

FIG. 5: Calibrating Bob's Longitudinal Light-Clock. (A short proof based on Mermin. $\cdot$ ) Interpreting triangle $O S F$ as a Doppler Effect (the period between reception events, $O$ and $F$, is proportional to the period between emission events, $O$ and $S$ ), we have $t_{F}^{(B o b)}=k t_{S}^{(\text {Alice) }}$, where $k$ is the Bondi-Doppler factor. Similarly, from triangle $O E T$, we have $t_{T}^{(\text {Alice) }}=k t_{E}^{(B o b)}$, with the same $k$-factor (in accordance with the Relativity Principle). By similar triangles, $t_{T}^{(\text {Alice) }} / t_{S}^{(\text {Alice })}=v_{T} / v_{F}$ and $t_{F}^{(B o b)} / t_{E}^{(B o b)}=u_{F} / u_{T}$. When $t_{T}^{(\text {Alice })}=t_{F}^{(B o b)}$, it follows that $u_{T} v_{T}=u_{F} v_{F}$. Geometrically, this means the required parallelograms $O M T N$ and $O Y F Z$ have equal areas, and that events $T$ and $F$ lie on the hyperbola $u v=\left(t_{T}^{(\text {Alice })}\right)^{2}$ with asymptotes along $O$ 's light cone.

Refer now to Fig. 5, Following Mermin,,$\frac{5}{,}$ let us describe the sides of the causal diamonds with Alice's light-cone coordinates $v_{T}, v_{F}, u_{T}$, and $u_{F}$ so that we have the "areas" $25 u_{T} v_{T}$ and $u_{F} v_{F}$ for Alice's and Bob's causal diamonds. By similar triangles, we have

$$
\frac{t_{T}^{(\text {Alice })}}{t_{S}^{(\text {Alice })}}=\frac{v_{T}}{v_{F}} \quad \text { and } \quad \frac{t_{F}^{(B o b)}}{t_{E}^{(B o b)}}=\frac{u_{F}}{u_{T}} .
$$

Forming the ratio $(O F) /(O T)$, we use Eq. (15) (Doppler) with Eq. (8) (Relativity) and Eq. (9) (similiar triangles) to obtain

$$
\frac{t_{F}^{(B o b)}}{t_{T}^{(\text {Alice })}}=\frac{t_{F}^{(B o b)}}{k t_{E}^{(B o b)}}=\frac{u_{F}}{k u_{T}} .
$$


Similarly, using Eq. (7) with Eq. (8) and Eq. (9) we have

$$
\frac{t_{F}^{(\text {Bob })}}{t_{T}^{(\text {Alice })}}=\frac{k t_{S}^{(\text {Alice })}}{t_{T}^{(\text {Alice })}}=\frac{k v_{F}}{v_{T}},
$$

Upon multiplication of Eqs. (10) and (11), we eliminate the $k$-factors to obtain

$$
\left(\frac{t_{F}^{(B o b)}}{t_{T}^{(\text {Alice })}}\right)^{2}=\frac{u_{F} v_{F}}{u_{T} v_{T}}
$$

where the right-hand side is the ratio of areas of two causal diamonds. This means that, in units of the area of Alice's light-clock diamond, the area of the causal diamond of OF is equal to the square-interval of segment $O F$. To locate the event $F$ that marks Bob's first tick, we require $t_{F}^{(\text {Bob) }} / t_{T}^{(\text {Alice })}=1$. Thus, event $F$ is located at the opposite corner of a causal diamond with diagonal along $O V$ and with area equal to that of Alice's light-clock diamond (along $O T$ ).

Now that we have constructed Bob's tick, three relativistic effects are immediately evident from the spacetime diagram (Fig. [5). Using Alice's ticks, we find that the elapsed-time for Bob's tick OF is larger than that of her own tick OT. This is the time-dilation effect. Although Alice's reflection events are simultaneous according to Alice, Bob's reflection events are not simultaneous according to Alice. This is the relativity of simultaneity. Upon constructing the worldlines of the mirrors for Bob's light-clock (as we did in Fig. 4), Alice determines that the length of Bob's light-clock is shorter than her identically constructed light-clock. This is the length-contraction effect.

Note that when $t_{F}^{(\text {Bob) }} / t_{T}^{(\text {Alice })}=1$, Eqs. (10) and (11) can be rewritten as

$$
\begin{aligned}
& u_{F}=k u_{T} \\
& v_{F}=\frac{1}{k} v_{T},
\end{aligned}
$$

which represent an area-preserving transformation of Alice's light-clock diamond into Bob's light-clock diamond, by stretching along the $u$-direction by a factor $k$ and shrinking along the $v$-direction by a factor $k$-that is, an aspect ratio $u_{F} / v_{F}=k^{2}$. Physically, this preserves the speed of light (since the slopes of light-rays are unchanged) and the square-interval of the diagonal of the light-clock diamond (since $u_{F} v_{F}=u_{T} v_{T}$ ). This is an active Lorentz boost 
transformation, expressed in a basis of its lightlike eigenvectors, with eigenvalues $k$ and $1 / k$. To recover the standard rectangular form, write half of the sum and half of the difference of Eqs. (13) and (14), then use Eq. (3) and Eq. (6). (See Eq. (A.4) in the Appendix for a similar calculation for the passive Lorentz Boost coordinate transformation.)

\section{B. A construction for use with students}

In this section, we construct Bob's ticks using an alternate method that has been used with students. Our story uses radar-experiments and the Doppler Effect (in the spirit of the Bondi $k$-calculus $\frac{11}{}$ method) framed in the context of television transmissions sent and received by Alice and Bob. $\underline{26}$

With a sheet of rotated graph paper, we draw Alice's worldline and her light-clock diamonds. (See Fig. 3.) While the computer-generated shading is useful for visualization, it is not a necessary feature for visual calculations. Using Alice's light-clock diamonds, we draw Bob's worldline along $O J$ with velocity $\beta=(3 / 5)$. We construct two segments, $O H$ along Alice's worldline and $H J$ where $H$ and $J$ are simultaneous according to Alice, such that $(H J) /(O H)=(3 / 5)$. We now begin the construction of Bob's light-clock diamonds.

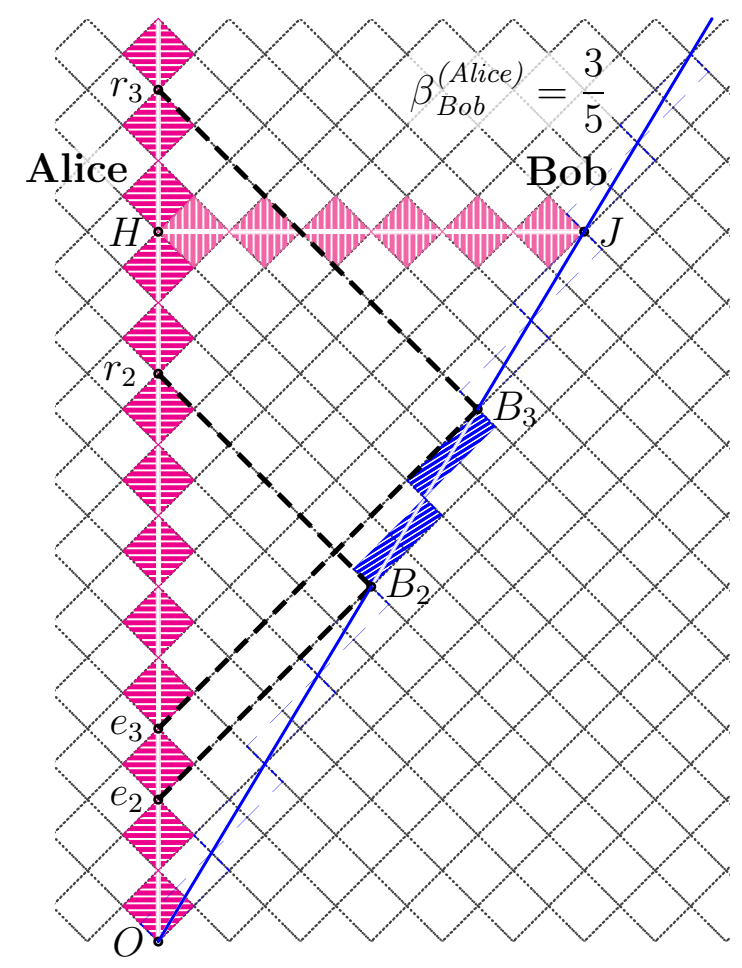

FIG. 6: Calibrating Bob's Longitudinal Light-Clock. (Motivation for students.) 
Alice sends to Bob two successive light-signals, the second signal sent one tick after the first. We interpret these as broadcasts marking the start and end of a one-hour television program produced by Alice. Due to the finiteness of the speed of light, Bob receives the first signal after a delay. However, since Bob is receding from Alice, Bob receives the second signal after $k$ of his ticks, where $k$ is a proportionality constant to be determined. That is,

$$
\Delta t_{\text {receptions }}^{(\text {Bob })}=k \Delta t_{\text {emissions }}^{(\text {Alice }}
$$

(In the previous section, the constant $k$ was called $k_{\text {Alice }}^{(B o b)}$.) It is easy to see that $k=1$ for a distant observer at rest according to Alice, but $k>1$ for a distant inertial observer who is receding from Alice. Thus, Bob would take $k$ of his hours to watch 27 in slow-motion a one-hour program by Alice.

In order to determine $k$, we arrange to have Bob immediately rebroadcast the received signals from Alice. By the Relativity Principle, Alice must receive the delayed broadcast from Bob at the same slowed rate. Alice would take $k$ of her hours to watch in slow-motion a one-hour program by Bob. So,

$$
\begin{aligned}
\Delta t_{\text {receptions }}^{(\text {Alice })} & =k \Delta t_{\text {emissions }}^{(\text {Bob })} \\
& =k\left(\Delta t_{\text {receptions }}^{(\text {Bob })}\right)=k\left(k \Delta t_{\text {emissions }}^{(\text {Alice })}\right)
\end{aligned}
$$

Thus, Alice would take $k^{2}$ of her hours to watch in very slow-motion her originally broadcasted one-hour program. By counting Alice's light-clock diamonds off the spacetime diagram, one can determine, for $\beta=(3 / 5)$, the corresponding value of $k^{2}$ :

$$
k^{2}=\frac{\Delta t_{\text {receptions }}^{(\text {Alice })}}{\Delta t_{\text {emissions }}^{(\text {Alice })}}=\frac{t_{r_{3}}^{(\text {Alice })}-t_{r_{2}}^{(\text {Alice })}}{t_{e_{3}}^{(\text {Alice })}-t_{e_{2}}^{(\text {Alice })}}=4,
$$

and thus determine $k=2$. So, there must two of Bob's clock-ticks between events $B_{2}$ and $B_{3}$ on his worldline. Since the sides of the light-clock diamonds are traced out by light-signals (drawn parallel to the lines of the rotated grid), one is led to drawing two congruent causal diamonds, each a prototype of Bob's light-clock diamond.

We then ask the student to somehow characterize Bob's light-clock diamond so as to avoid having to always do a radar-experiment to construct it. By being able to count 
squares on the rotated grid, it is realized that Bob's light-clock diamond has the same area as Alice's. Indeed, the edges of Bob's light-clock diamond have sizes $k$ and $1 / k$, compared to the corresponding edges of Alice's light-clock diamond. We can now draw Bob's light-clock diamonds along his entire worldline.

Furthermore, the student is led to notice that the causal diamond from $B_{2}$ to $B_{3}$ (two events on Bob's worldline) has the same shape as one of Bob's light-clock diamonds, and that the area of that causal diamond is equal to the square of the proper-time interval from $B_{2}$ to $B_{3}$. Note that both Alice and Bob, each using their light-clock diamonds can partition the area of that causal diamond into $k^{2}=4$ light-clock diamond areas, which also displays the invariance of the square-interval.

These conclusions can be reinforced by repeating the construction for another numerically convenient velocity, say, $\beta=(4 / 5)$.

\section{Bob's Coordinate Systems}

With the prototype for Bob's light-clock diamonds, it is now easy to construct Bob's rectangular and light-cone coordinate systems, in complete analogy to Alice's coordinate systems. (Refer to Figures 7 and 8 and their captions, and compare with Figures 2 and 3 .)

As mentioned in Sec. IV A, three relativistic effects are evident from the diagram. We will discuss the time-dilation and length-contraction effects in the Applications (Sections $\mathrm{VA}$, $\mathrm{VB}$, and $\mathrm{VD}$ ). Here, we address the remaining effect.

A striking feature of Bob's rectangular coordinate system (in Fig. 7) is that Bob's $x$-axis is not parallel with Alice's $x$-axis. Since an observer's $x$-axis represents a set of events that are simultaneous with the event at $t=0$ for that observer, the spacetime diagram indicates that these observers will disagree on whether two distinct events (say, events $O$ and $G$ ) are simultaneous. This is the relativity of simultaneity.

A related feature is that Bob's $x$-axis is not perpendicular-in the familiar Euclidean sense - to Bob's $t$-axis, as it appears to be for Alice's $x$ - and $t$-axes. ${ }^{28}$ This feature is an indication that the geometry of this spacetime diagram is not a Euclidean geometry, but a Minkowskian geometry. ${ }^{2}$ One important difference is that the notion of perpendicularity in a spacetime diagram is not specified by a circle, but by a causal diamond (which encodes some

properties of the Minkowskian analogue of a circle, the hyperbola). From the discussion in 
Sec. III, since Bob's $x$ - and $t$-axes are parallel to the diagonals of some causal diamond (specifically, Bob's light-clock diamond), those axes are in fact "spacetime-perpendicular."

We will apply our construction to some examples from Special Relativity (in Sec. V) to help develop skills for reasoning physically and geometrically with spacetime diagrams.

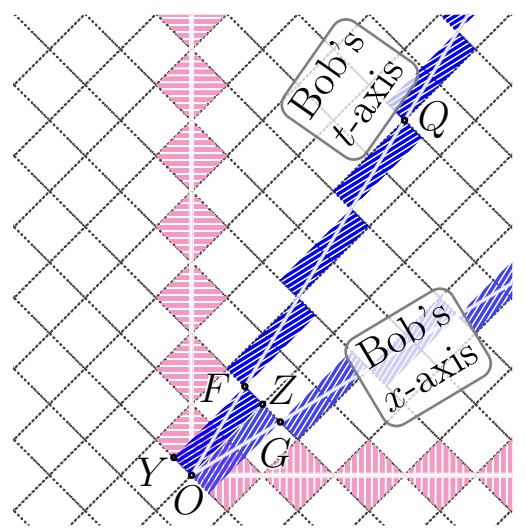

FIG. 7: Bob's Rectangular Coordinate System uses the diagonals of his light-clock diamonds to locate events. Bob's time-axis is marked by a string of light-clock diamonds with their timelikediagonals along $\overrightarrow{O F}$ on his worldline. Bob's spatial $x$-axis is marked by a string of light-clock diamonds with their spacelike-diagonals along $\overrightarrow{O G}$, on a line of simultaneity according to Bob (parallel to $Y Z$ ) through event $O$. From the diagram, since $\overrightarrow{O Q}=4 \overrightarrow{O F}+0 \overrightarrow{O G}$, Bob assigns event $Q$ rectangular coordinates $\left(t_{Q}^{(B o b)}, x_{Q}^{(B o b)}\right)=(4,0)$.

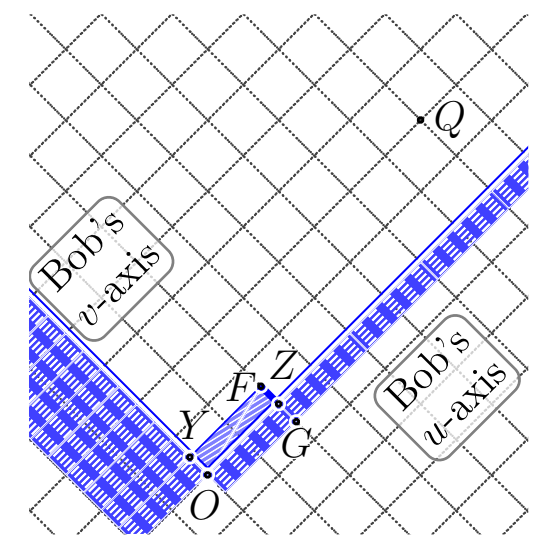

FIG. 8: Bob's Light-Cone Coordinate System uses the edges of his light-clock diamonds to locate events. Since $\overrightarrow{O F}=\overrightarrow{O Z}+\overrightarrow{O Y}$ and $\overrightarrow{O G}=\overrightarrow{O Z}-\overrightarrow{O Y}$, we have $\overrightarrow{O Q}=4 \overrightarrow{O Z}+4 \overrightarrow{O Y}$. So, Bob assigns event $Q$ light-cone coordinates $\left(u_{Q}^{(B o b)}, v_{Q}^{(B o b)}\right)=(4,4)$. Analogous to the situation with Alice, Bob's $u$ - and $v$-axes are marked by strings of light-clock diamonds with their lightlike-edges along $\overrightarrow{O Z}$ and $\overrightarrow{O Y}$, respectively, on the light-cone of event $O$. 


\section{Subdivided grids}

As we have seen, when the relative-velocity $\beta$ of an inertial observer is rational, say $\beta=\Delta x / \Delta t$ where $\Delta x$ and $\Delta t$ are integers, the worldline of that observer can be constructed by using a Minkowski-right triangle formed by counting off $\Delta t$ of Alice's temporally-arranged diamonds, followed by counting off $\Delta x$ of Alice's spatially-arranged diamonds. Since the square-interval of the hypotenuse is the integer $\Delta s^{2}=(\Delta t)^{2}-(\Delta x)^{2}$, the area of its causal diamond can be determined by counting grid squares.

To go further and count off an integer number $\Delta s$ of diamonds along the hypotenuse, the square-interval should be a perfect square. This occurs when the Bondi $k$-factor $k=$

$\sqrt{(1+\beta) /(1-\beta)}($ Eq. (6) $)$ ) is rational, with value $\Delta s /(\Delta t-\Delta x)$. (These restrictions can be formulated in terms of Pythagorean triples. $\frac{29}{}$ )

When the $k$-factors are ratios of small integers, it becomes easy to graphically construct the diamonds of the observers, especially if the grid is suitably subdivided. A $6 \times 6$ subdivision is useful since it can accomodate the $k$-factors $1,2,3 / 2$, and 3 and their reciprocals, which correspond to the velocities $0, \pm 3 / 5, \pm 5 / 13$, and $\pm 4 / 5$.

To handle arbitrary relative-velocities, one can advance to the use of algebraic formulas [derived later], whose meanings would have been motivated by the special cases noted above.

\section{APPLICATIONS}

We now offer a series of standard calculations in special relativity with our graphical method. These are concise summaries of what has been presented to students through a series of worksheets. Although we proceed in a sequence to highlight "relativistic effects," we regard the operational radar-measurement methods in Sec. VG to be a more fundamental starting point.

\section{A. Time dilation}

After leaving inertial observer Alice at event $O$, another inertial observer Bob travels at (3/5) according to Alice. Thus, according to Alice, after 5 of her ticks have elapsed, Bob is located 3 of her ticks away, at event $Q$. How much of Bob's proper time has elapsed from 
events $O$ to $Q$, both on his worldline?

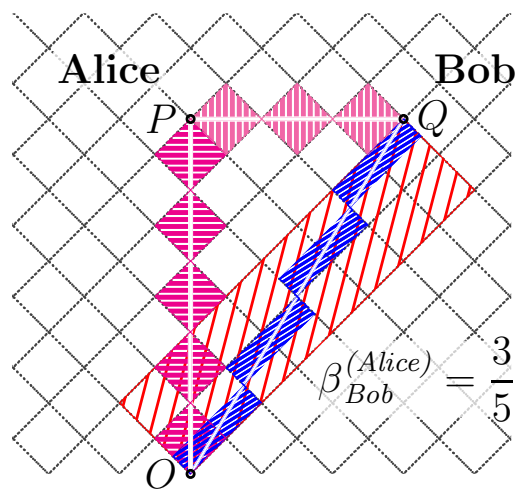

FIG. 9: Time Dilation: $t_{Q}^{(\text {Alice })}=t_{P}^{(\text {Alice })}>t_{Q}^{(\text {Bob })}$.

In Fig. 9, we use Alice's diamonds to draw Bob's worldline, with $O P$ and $P Q$ chosen so that $(P Q) /(O P)=\beta=3 / 5$. Since the causal diamond of $O Q$ has $O Q$ as the timelike diagonal, the proper-time elapsed along $O Q$ is equal to the square root of the area of that causal diamond. From the diagram, we count the area of the causal diamond to be 16, so that the proper-time is $\sqrt{16}=4$ ticks. We can now draw 4 of Bob's light-clock diamonds. Note that while Bob declares the elapsed time between the events $O$ and $Q$ that he experiences is 4 ticks, Alice declares the elapsed time to be 5 ticks. This is the time dilation effect, with timedilation factor $\gamma=(O P) /(O Q)=5 / 4$. This agrees with the formula $\gamma=\left(1-\beta^{2}\right)^{-1 / 2}=5 / 4$ for $\beta=(3 / 5)$, which we explicitly use in Sec. $\mathrm{VE}$, and derive in the Appendix.

More generally, in the timelike-future of $O$, consider two events, $P$ and $Q$, that Alice determines to be simultaneous. The ratio of the areas of the causal diamonds of $O P$ (not shown) and of $O Q$ is equal to $\gamma^{2}$, the square of the time-dilation factor.

\section{B. Symmetry of the Inertial Observers}

Refer to Figure 10. In accordance with the Relativity Principle, for two events, $O$ and $Q^{\prime}$, experienced by Alice, Bob will observe a longer time-interval between those events than Alice will - with the same time-dilation factor $\gamma=5 / 4$. Using Bob's diamonds, we construct the analogue of Alice's diagram. Since the small diagram we have been using becomes a little cluttered when displaying the symmetry, we have included additional pairs of events $\{H, J\}$ and $\left\{H^{\prime}, J^{\prime}\right\}$ in Fig. 10.

Note that the use of similarity in this diagram suggests that problems involving this 


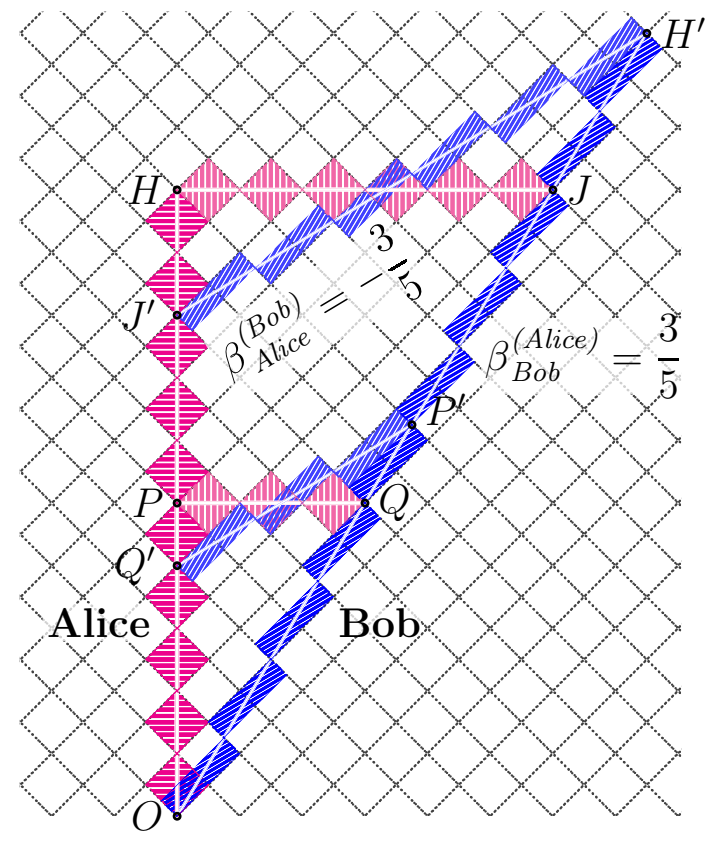

FIG. 10: Symmetry of time-dilation.

relative-speed with arbitrary lengths and time can be handled by setting up proportions.

\section{The Clock Effect}

After leaving Alice at event $O$, Bob travels away at velocity (3/5), instantaneously turning around at a distant event $Q$ and returning with velocity - (3/5). At the reunion event $Z$, if Alice had aged 10 ticks since the separation event, how much did Bob age?

Refer to Fig. 11, Determine Bob's diamonds by treating his non-inertial trip as piecewiseinertial segments. By constructing the causal diamonds from $O$ to $Q$, then from $Q$ to $Z$, we determine from the diagram $\sqrt{16}$ ticks along $O Q$, followed by another $\sqrt{16}$ diamonds along $Q Z$, for a total of 8 ticks. This does not equal the 10 ticks logged by Alice's [inertial] trip from $O$ to $Z$. This route-dependence of elapsed proper-time between two events is the Clock Effect.

\section{Length Contraction}

Bob traveling at $(3 / 5)$ carries a ladder that is 5 ticks long. How long is that ladder according to Alice?

Refer to Fig. 12, We use Alice's diamonds to draw Bob's worldline and his diamonds, with 


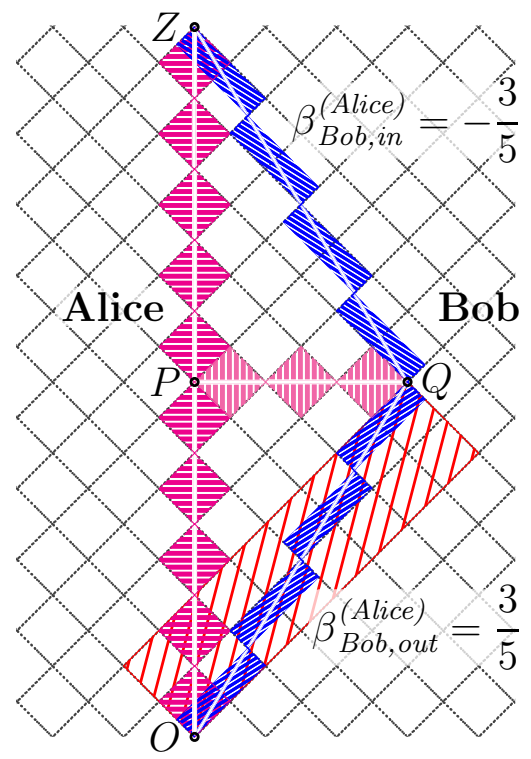

FIG. 11: Clock Effect. Alice ages 10 ticks along her inertial worldline from $O$ to $Z$, whereas Bob (traveling to and from event $Q$ with speed $(3 / 5)$ ) ages $4+4=8$ ticks along his non-inertial worldline from $O$ to $Q$ to $Z$.

$O P$ and $P Q$ chosen so that $(P Q) /(O P)=3 / 5$. Then, use Bob's light-clock diamonds to measure from event $O$ to event $Y$, a spatial displacement of 5 ticks, the ladder's rest-length. (Note that Bob regards events $O$ and $Y$ as simultaneous.) Through event $Y$, construct the worldline of the ladder's far end as a parallel to Bob's worldline.

Alice determines the length of Bob's ladder by finding the spatial distance between events $O$ and $X$, where $X$ is the event on the worldline of the ladder's far end that Alice regards as simultaneous with $O$. Since $O X$ is the spatial-diagonal of the causal diamond between $O$ and $X$, the length of the ladder according to Alice is square-root of the area of the causal diamond between $O$ and $X:(O X)=4$ ticks. Since $(O X)<(O Y)$, the observed-length of the ladder is shorter than its rest-length. This is the length-contraction effect, with length-contraction factor equal to the time-dilation factor $\gamma=5 / 4$.

By symmetry, Bob determines the length of Alice's identical ladder, with its far end along a worldline (not shown) through events $X^{\prime}$ and $Y^{\prime}$, to be shorter than its rest-length by the same length-contraction factor. [A triangle like $O X^{\prime} Y^{\prime}$ will be used in a proof for the Lorentz Boost transformation formula shown in Fig. 14.] 


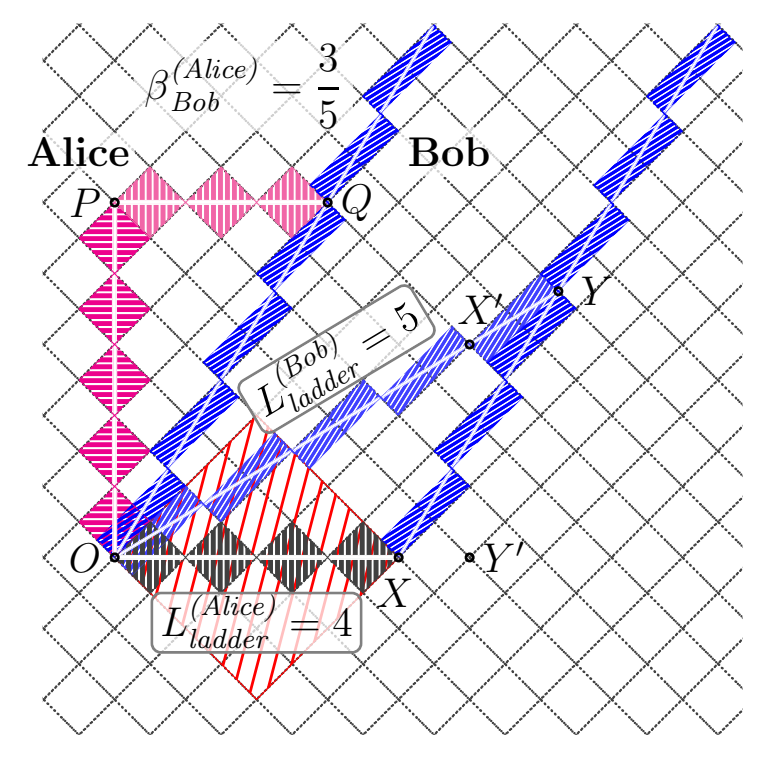

FIG. 12: Length Contraction: $L_{\text {ladder }}^{(\text {Alice })}<L_{\text {ladder }}^{(\text {Bob })}$

\section{E. Lorentz Boost coordinate transformation}

Bob, traveling with velocity (3/5) according to Alice, assigns event E coordinates $\left(t_{E}^{(B o b)}, x_{E}^{(B o b)}\right)=(2,6)$. What coordinates would Alice assign to E? (Both Alice and Bob regard their meeting event $O$ as the origin of their coordinates.)

Refer to Fig. 13. As before, we use Alice's diamonds to draw Bob's worldline and his diamonds. With Bob's diamonds, we locate the event E. Now, using Alice's damonds, we find $\left(t_{E}^{\text {(Alice) }}, x_{E}^{(\text {Alice })}\right)=(7,9)$. This agrees with the result from the Lorentz Boost coordinate transformation formulae (derived in Fig. 14 and in the Appendix):

$$
\begin{aligned}
& t_{E}^{(\text {Alice })}=\gamma\left(t_{E}^{(\text {Bob })}+\beta_{\text {Bob }}^{(\text {Alice })} x_{E}^{(\text {Bob })}\right)=\frac{5}{4}\left(2+\frac{3}{5} 6\right)=7 \\
& x_{E}^{(\text {Alice })}=\gamma\left(x_{E}^{(\text {Bob })}+\beta_{\text {Bob }}^{(\text {Alice })} t_{E}^{(\text {Bob })}\right)=\frac{5}{4}\left(6+\frac{3}{5} 2\right)=9
\end{aligned}
$$

where $\gamma=5 / 4$ is the time-dilation factor (introduced in Sec. $\overline{\mathrm{VA}}$ ) for velocity $\beta_{\text {Bob }}^{(\text {Alice }}=(3 / 5)$.

In addition, note that the causal diamond with $O E$ as its spacelike diagonal has area 32, which is equal to minus the square-interval of $O E,-\left((2)^{2}-(6)^{2}\right)=-\left((7)^{2}-(9)^{2}\right)$. We will elaborate on this point in Section VG.

We can use the diagram to derive the inverse Lorentz Boost coordinate transformation. Refer to Fig. 14. (A similar derivation appears in Ellis. ${ }^{20}$ ) 


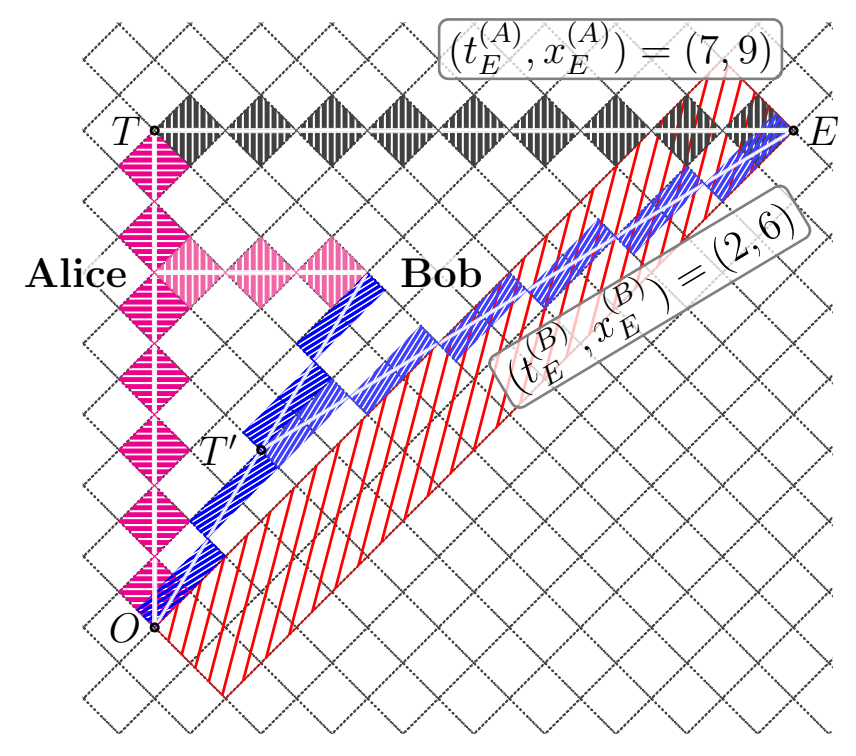

FIG. 13: Comparison of coordinates without the Lorentz Boost coordinate transformation.

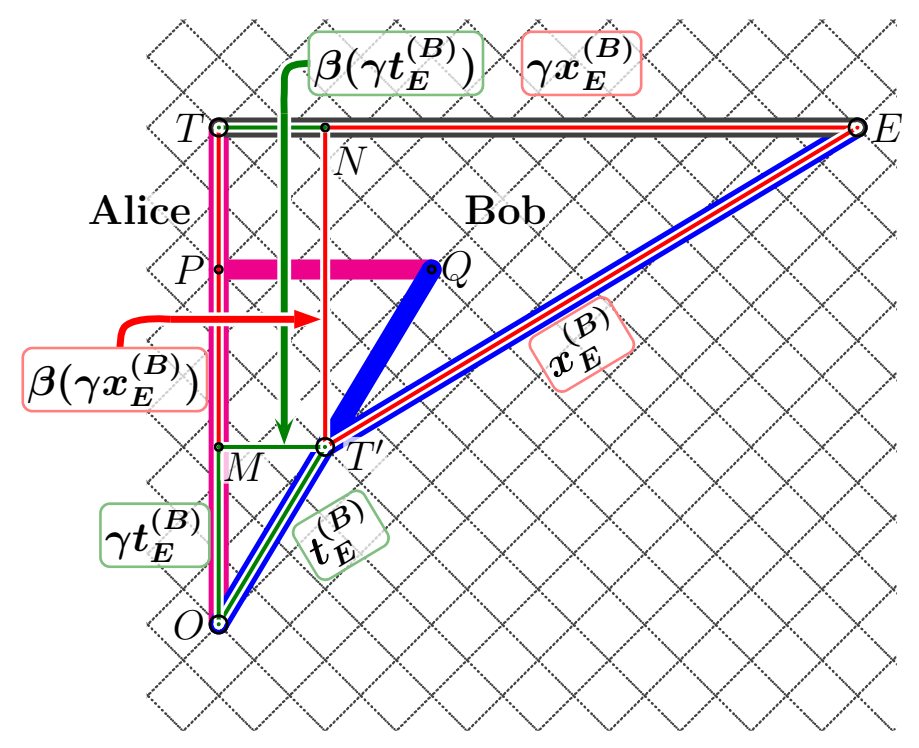

FIG. 14: A geometrical derivation of the Lorentz Boost coordinate transformation. Since $\gamma=$ $(O P) /(O Q)=(O M) /\left(O T^{\prime}\right)$ and $\gamma=(E N) /\left(E T^{\prime}\right)$ and $\beta=(P Q) /(O P)=\left(M T^{\prime}\right) /(O M)$ and $\beta=(E N) /\left(N T^{\prime}\right)$, we have $t_{E}^{(\text {Alice })}=(O M)+(M T)=(O M)+\left(T^{\prime} N\right)=\left(\gamma t_{E}^{(B o b)}\right)+\beta\left(\gamma x_{E}^{(B o b)}\right)$ and $x_{E}^{(\text {Alice })}=(T N)+(N E)=\left(M T^{\prime}\right)+(N E)=\beta\left(\gamma t_{E}^{(B o b)}\right)+\gamma x_{E}^{(B o b)}$. A similar derivation appears in Ellis 20

\section{F. Velocity Composition}

Alice, Bob, and Carol met briefly at event O. Bob, traveling with velocity (3/5) according to Alice, observes Carol moving forward with velocity (5/13). What is the velocity of Carol according to Alice? 
Refer to Fig. 15. Alice uses her diamonds to draw Bob's worldline and his diamonds, with $O P$ and $P Q$ chosen so that $(P Q) /(O P)=3 / 5$. Next, using Bob's diamonds, construct Carol's worldline along $O X$, with $O X$ and $X Y$ chosen so that $(X Y) /(O X)=5 / 13$. Alice, using her diamonds again, determines the slope of Carol's worldline $O Y$ by choosing an event $T$ on her worldline that she regards as simultaneous with $Y$. Then, the velocity of Carol according to Alice is determined by $(T Y) /(O T)$. From the graph paper, we determine $\beta_{\text {Carol }}^{\text {(Alice) }}=(16 / 20)=(4 / 5)$. This agrees with the result obtained by the velocity composition formula:

$$
\begin{aligned}
\beta_{\text {Carol }}^{(\text {Alice })} & =\frac{\beta_{\text {Bob }}^{(\text {Alice })}+\beta_{\text {Carol }}^{(\text {Bob })}}{1+\beta_{\text {Bob }}^{(\text {Alice })} \beta_{\text {Carol }}^{(\text {Bob })}} \\
& =\frac{\frac{3}{5}+\frac{5}{13}}{1+\left(\frac{3}{5}\right)\left(\frac{5}{13}\right)}=\frac{4}{5}
\end{aligned}
$$

(Proof: Write $\beta_{\text {Carol }}^{(\text {Alice })}=x_{Y}^{(\text {Alice) }} / t_{Y}^{(\text {Alice })}$, then substitute the analogues of Eq. (19) and Eq. (18). Since $x_{Y}^{(B o b)}=\beta_{\text {Carol }}^{(B o b)} t_{Y}^{(B o b)}$, the velocity composition formula follows. A similar derivation appears in Taylor. $\underline{16}^{-}$An alternate proof based on radar-methods is given in the Appendix.)

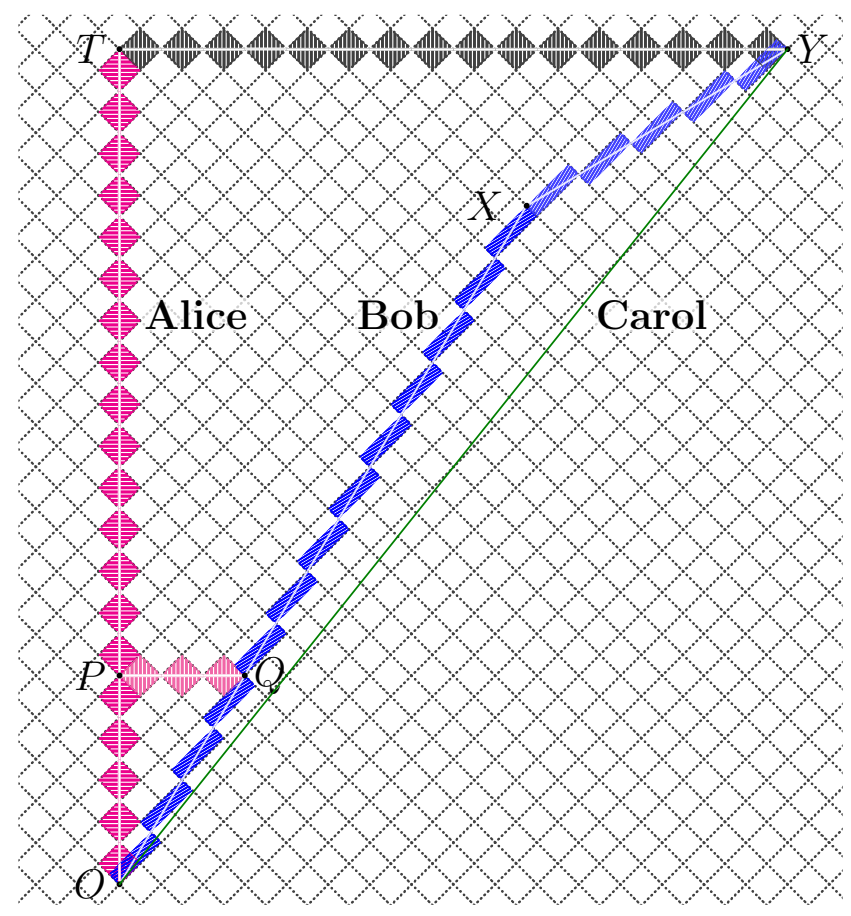

FIG. 15: Velocity Composition 


\section{G. Radar Measurements and the Invariant Square-Interval}

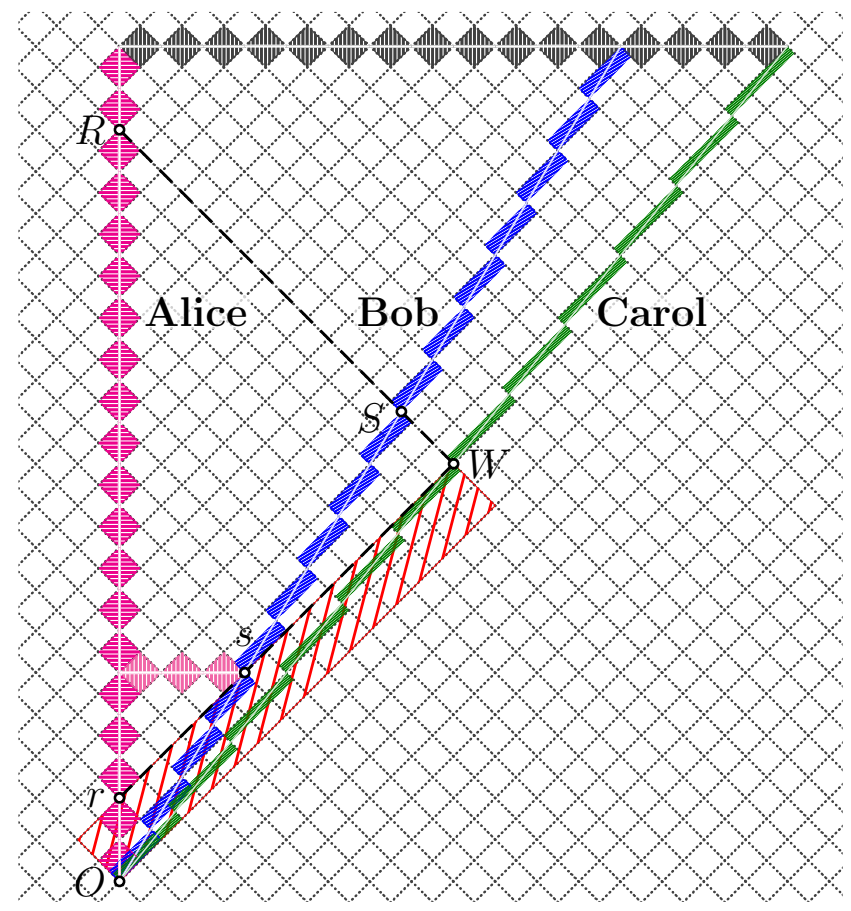

FIG. 16: Radar Measurements of event $W$.

We reuse parts of the previous situation in Sec. VF to describe radar measurements. (Refer to Fig. 16.) In this section, note the many interesting kinematical quantities that can be expressed in terms of the counting of clock ticks on an observer's worldline $\underline{\underline{11}-15} \underline{\underline{19}-21}$ We feel that this is a more natural starting point for the development of the subject.

Alice operationally assigns coordinates to event $W$ using a radar-measurement, emitting a light-signal at event $r$ to the target event $W$, then receiving its echo at event $R$. From the two elapsed times (since the origin event $O$ ) on her worldline, $t_{r}^{(\text {Alice })}$ and $t_{R}^{(\text {Alice })}$. Alice's rectangular coordinates for $W$ are obtained by

$$
t_{W}^{(\text {Alice })}=\frac{1}{2}\left(t_{R}^{(\text {Alice })}+t_{r}^{(\text {Alice })}\right) \quad \text { and } \quad x_{W}^{(\text {Alice })}=\frac{1}{2}\left(t_{R}^{(\text {Alice })}-t_{r}^{(\text {Alice })}\right),
$$

which can be interpreted as the "midway time between emission and reception" and "half of the round-trip distance," respectively. From the diagram, Alice measures $t_{r}^{(\text {Alice })}=2$ and $t_{R}^{(\text {Alice })}=18$, so that $t_{W}^{(\text {Alice })}=10$ and $x_{W}^{(\text {Alice })}=8$. Similarly, for Bob, using events $s$ and $S$,

$$
t_{W}^{(B o b)}=\frac{1}{2}\left(t_{S}^{(B o b)}+t_{s}^{(B o b)}\right) \quad \text { and } \quad x_{W}^{(B o b)}=\frac{1}{2}\left(t_{S}^{(B o b)}-t_{s}^{(B o b)}\right),
$$


with $t_{s}^{(B o b)}=4$ and $t_{S}^{(B o b)}=9$, so that $t_{W}^{(B o b)}=6.5$ and $x_{W}^{(B o b)}=2.5$.

In general, Alice and Bob disagree on their $t$ - and $x$-coordinates for $W$. However, they agree on the invariant square-interval $\left(t_{W}\right)^{2}-\left(x_{W}\right)^{2}$, which can be expressed as the product of their radar times: $12,15,19$

$$
\left(t_{W}\right)^{2}-\left(x_{W}\right)^{2}=t_{R}^{(\text {Alice })} t_{r}^{(\text {Alice })}=t_{S}^{(\text {Bob })} t_{s}^{(\text {Bob })}=36 .
$$

Let us observe that, for each inertial observer, the pair of radar times correspond to that observer's light-cone coordinates ${ }^{3}$ of event $W$. From Eq. (3), we have $u \equiv t+x$ and $v \equiv t-x$. Since events $r$ and $R$ are on Alice's worldline (where $\left.x_{r}^{(\text {Alice })}=0\right), u_{r}^{(\text {Alice })}=v_{r}^{(\text {Alice })}=t_{r}^{(\text {Alice) }}$ and $u_{R}^{(\text {Alice })}=v_{R}^{(\text {Alice })}=t_{R}^{(\text {Alice })}$. Refer again to Fig. 16. Since $W$ is on the past-light-cone of event $R$ and $x_{W}^{(\text {Alice })}>0$, we have $u_{W}^{(\text {Alice })}=u_{R}^{(\text {Alice })}$. Since $W$ is on the future-light-cone of event $r$ and $x_{W}^{(\text {Alice })}>0$, we have $v_{W}^{(\text {Alice })}=v_{r}^{(\text {Alice })}$. Thus,

$$
\left(u_{W}^{(\text {Alice })}, v_{W}^{(\text {Alice })}\right)=\left(t_{R}^{(\text {Alice })}, t_{r}^{(\text {Alice })}\right)
$$

Similarly,

$$
\left(u_{W}^{(B o b)}, v_{W}^{(B o b)}\right)=\left(t_{S}^{(B o b)}, t_{s}^{(B o b)}\right) .
$$

Furthermore, this means that the invariant square-interval in Eq. (23) can be written in light-cone coordinates as

$$
u_{W}^{(\text {Alice })} v_{W}^{(\text {Alice })}=u_{W}^{(B o b)} v_{W}^{(\text {Bob })}=36
$$

which is equal to the area of the causal diamond of $O W$. For Carol (along $O W$ ), her radar experiment for $W$ has her emission and reception events coinciding with the target event $W$. Thus, $\left(t_{W}^{(\text {Carol })}\right)^{2}=36$, which means that there are $\sqrt{36}=6$ of Carol's light-clock diamonds from event $O$ to event $W$.

From the rectangular coordinates, one can operationally determine the relative velocities of the inertial observers by, for example,

$$
\beta_{\text {Carol }}^{(\text {Alice })}=\frac{x_{W}^{(\text {Alice })}-x_{O}^{(\text {Alice })}}{t_{W}^{(\text {Alice })}-t_{O}^{(\text {Alice })}}=\frac{\left(t_{R}^{(\text {Alice })}-t_{r}^{(\text {Alice })}\right)-0}{\left(t_{R}^{(\text {Alice })}+t_{r}^{(\text {Alice })}\right)-0} .
$$


This velocity can be related to the $k$-factors first introduced in Sec. IV. Applying the idea behind Eq. (17), we have $\left(k_{\text {Alice }, \text { Carol }}\right)^{2}=t_{R}^{(\text {Alice })} / t_{r}^{(\text {Alice })}$. Thus,

$$
\beta_{\text {Carol }}^{\text {(Alice })}=\frac{\left(\frac{\left(t_{R}^{(\text {Alice })}\right)}{t_{r}^{(\text {Alice })}}\right)-1}{\left(\frac{\left(t_{R}^{(\text {Alice })}\right)}{t_{r}^{(\text {Alice })}}\right)+1}=\frac{\left(k_{\text {Alice }, \text { Carol }}\right)^{2}-1}{\left(k_{\text {Alice }, \text { Carol }}\right)^{2}+1} .
$$

Upon solving that equation for $k_{\text {Alice,Carol, }}$, we can recognize it as the familiar Doppler factor

$$
k_{\text {Alice }, \text { Carol }}=\sqrt{\frac{1+\beta_{\text {Carol }}^{(\text {Alice })}}{1-\beta_{\text {Carol }}^{(\text {Alice })}}},
$$

which we had given earlier (without proof) as Eq. (6).

With Alice's measurements of event $W$ obtained earlier, $t_{r}^{(\text {Alice })}=2$ and $t_{R}^{(\text {Alice })}=18$, we have $k_{\text {Alice }, \text { Carol }}=\sqrt{t_{R}^{(\text {Alice })} / t_{r}^{(\text {Alice })}}=\sqrt{18 / 2}=3$ and $\beta_{\text {Carol }}^{\text {Alice }}=(18-2) /(18+2)=4 / 5$. Similarly, with Bob's measurements of $t_{s}^{(B o b)}=4$ and $t_{S}^{(B o b)}=9$, we have $k_{B o b, C a r o l}=$ $\sqrt{t_{S}^{(B o b)} / t_{s}^{(B o b)}}=\sqrt{9 / 4}=3 / 2$ and $\beta_{\text {Carol }}^{(B o b)}=(9-4) /(9+4)=5 / 13$. These are consistent with the velocity composition analysis in Sec. $\mathrm{VF}$.

At this point, one could go further in this viewpoint to derive the velocity composition formula and the Lorentz Boost coordinate transformations. We do this in the Appendix.

\section{H. Collisions in Energy-Momentum Space}

In addition to kinematical examples in spacetime, this method can be applied to collision problems in energy-momentum space. Analogous to the causal diamond of a timelike displacement, let us consider the "mass diamond" of a timelike momentum-vector, whose area is equal to the square of the associated rest-mass. The lengths of momentum-vectors can now be described by tickmarks of mass-units.

In the energy-momentum diagram of Fig. 17, we describe an elastic collision ${ }^{30}$ of two particles with rest-masses $m_{1}=12$ and $m_{2}=8$, initial-velocities $\beta_{1, i}=-5 / 13$ and $\beta_{2, i}=$ 15/17, and final-velocities $\beta_{1, f}=4 / 5$ and $\beta_{2, f}=-3 / 5$. From the mass diamond (of size 40 by 20 with Alice's diamonds), we have $k_{\text {Alice } C O M}{ }^{2}=(40 / 20)=2$ so that the velocity of the center-of-momentum frame is $\beta_{C O M}^{(\text {Alice })}=(2-1) /(2+1)=1 / 3$ and the magnitude of the total- 


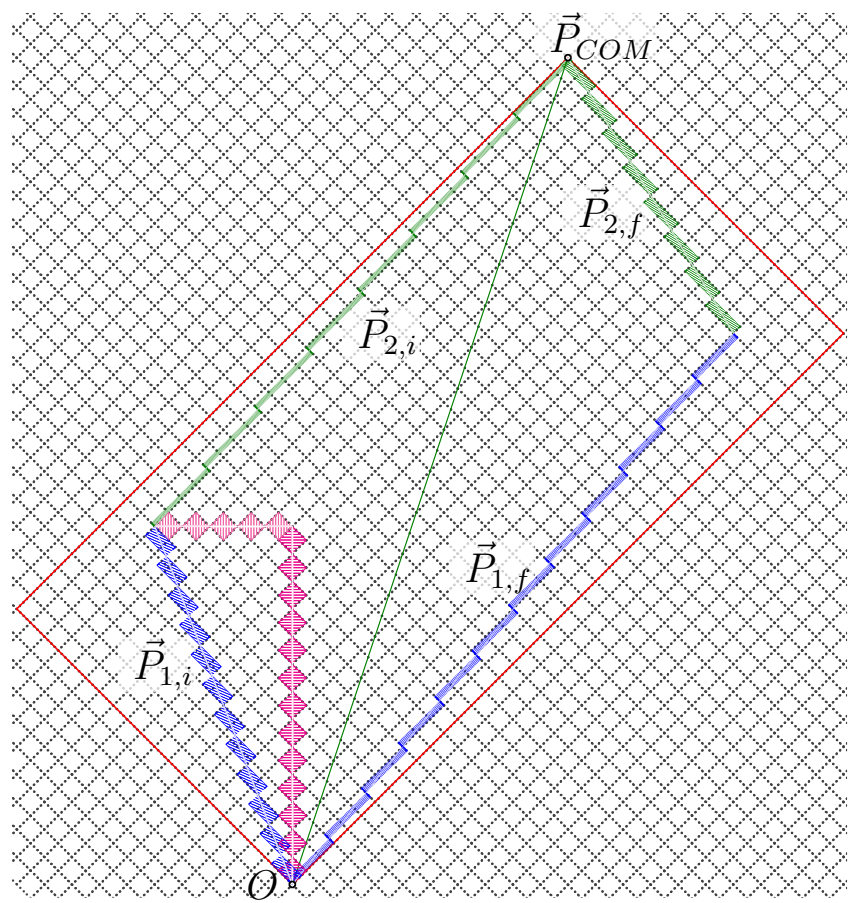

FIG. 17: An elastic collision (with $\vec{P}_{1, i}+\vec{P}_{2, i}=\vec{P}_{1, f}+\vec{P}_{2, f}$ ) drawn on Alice's energy-momentum diagram. The particles have rest-masses $m_{1}=12$ and $m_{2}=8$, initial-velocities $\beta_{1, i}=-5 / 13$ and $\beta_{2, i}=15 / 17$, and final-velocities $\beta_{1, f}=4 / 5$ and $\beta_{2, f}=-3 / 5$. We have drawn a "mass diamond" for the total momentum vector, $\vec{P}_{C O M}$.

momentum (called the "invariant mass" of the system of particles) is $P_{C O M}=\sqrt{(40)(20)}$. Alternatively, one can construct Alice's right-triangle with $P_{C O M}$ as its hypotenuse and determine $\beta_{C O M}^{\text {(Alice) }}=10 / 30$ and $P_{C O M}=\sqrt{(30)^{2}-(10)^{2}}$.

\section{THE AREA OF A CAUSAL DIAMOND, ALGEBRAICALLY}

In the geometric construction of Bob's light-clock diamonds, the key feature we exploited was the equality of the areas of Alice's and Bob's light-clock diamonds, which is due to the area-preserving property of the Lorentz Boost transformations (Eqs. (13) and (14)). However, for the visualization of this equality and subsequent reduction of algebraic computations to counting, we implicitly exploited the equality of the areas of a figure in a Minkowski spacetime diagram and in a Euclidean space. In addition, we have not directly addressed the units involved in our construction. We address these issues now with an algebraic formulation.

Consider Alice's light-clock diamond (the causal diamond of OT representing 1 tick of 
Alice's clock). The area (denoted by $\overrightarrow{\mathcal{A}}(O T)$ ) of Alice's diamond is obtained by computing the cross product of its edges $\overrightarrow{O N} \times \overrightarrow{O M}$. Using Eq. (2), we see that $\overrightarrow{\mathcal{A}}(O T)=\overrightarrow{O N} \times \overrightarrow{O M}=$ $\frac{1}{2} \overrightarrow{O X} \times \overrightarrow{O T}$, which can be interpreted as half of the cross product of the diamond's diagonals. Making use of either the Minkowski metric (so $\overrightarrow{O T} \cdot \overrightarrow{O T}=1, \overrightarrow{O X} \cdot \overrightarrow{O X}=-1$, and $\overrightarrow{O T} \cdot \overrightarrow{O T}=1$ ) or the usual Euclidean metric, we determine that $\frac{1}{2} \overrightarrow{O X} \times \overrightarrow{O T}$ has "size" $\frac{1}{2}$ tick" $^{2}$. (Although square-norms of this cross product may disagree in sign between the two metrics [depending on signature conventions], they fortunately agree in absolute value.) Since this factor of $\frac{1}{2}$ will complicate our counting calculations, we have chosen the units of diamond-area to be "the area of Alice's light-clock diamond," or simply "diamond" (as we have done earlier), rather than tick ${ }^{2}$.

In addition, since $\overrightarrow{\mathcal{A}}$ carries the units of area, the coordinates $(t, x)$ and $(u, v)$ must be dimensionless, as we declared in Sec. IIIB, (Had we written $t_{T}=1$ tick and $x_{T}=0$ ticks, so that $u_{T}=1$ tick and $v_{T}=1$ tick, we would have $u_{T} v_{T}=1$ tick $^{2}$ for the "area." However, we just found that the area of Alice's diamond is only $\frac{1}{2}$ tick $^{2}$.)

To incorporate all of these issues, we will perform an algebraic computation of the squareinterval of $O Q$ in Figs. 2 and 3 as an area. Recall Eq. (1):

$$
\overrightarrow{O Q}=t_{Q} \overrightarrow{O T}+x_{Q} \overrightarrow{O X}=u_{Q} \overrightarrow{O N}+v_{Q} \overrightarrow{O M}
$$

We now show that $\overrightarrow{\mathcal{A}}(O Q)$, the area of the causal diamond of $O Q$, is equal to the squareinterval of $O Q$ in units of $\overrightarrow{\mathcal{A}}(O T)$, the area of Alice's diamond. Starting with the edges of the causal diamond, we have

$$
\begin{aligned}
\overrightarrow{\mathcal{A}}(O Q) & \stackrel{?}{=} \overrightarrow{\mathcal{A}}(O T)(\overrightarrow{O Q} \cdot \overrightarrow{O Q}) \\
u_{Q} \overrightarrow{O N} \times v_{Q} \overrightarrow{O M} & \stackrel{?}{=} \overrightarrow{\mathcal{A}}(O T)\left(t_{Q} \overrightarrow{O T}+x_{Q} \overrightarrow{O X}\right) \cdot\left(t_{Q} \overrightarrow{O T}+x_{Q} \overrightarrow{O X}\right) \\
u_{Q} v_{Q} \overrightarrow{O N} \times \overrightarrow{O M} & \stackrel{?}{=} \overrightarrow{\mathcal{A}}(O T)\left(t_{Q}{ }^{2} \overrightarrow{O T} \cdot \overrightarrow{O T}+x_{Q}{ }^{2} \overrightarrow{O X} \cdot \overrightarrow{O X}+2 t_{Q} x_{Q} \overrightarrow{O T} \cdot \overrightarrow{O X}\right) \\
\left(t_{Q}+x_{Q}\right)\left(t_{Q}-x_{Q}\right) \overrightarrow{\mathcal{A}}(O T) & \stackrel{\vee}{=} \overrightarrow{\mathcal{A}}(O T)\left(t_{Q}{ }^{2}-x_{Q}{ }^{2}\right)
\end{aligned}
$$

where we have used Eq. (3) and the Minkowski metric. Alternatively, we can start with the diagonals of the causal diamond, $\overrightarrow{O Q}$ and $\overrightarrow{O Q}_{\perp}=u_{Q} \overrightarrow{O N}-v_{Q} \overrightarrow{O M}$, then compute $\overrightarrow{\mathcal{A}}(O T)=\frac{1}{2} \overrightarrow{O Q}_{\perp} \times \overrightarrow{O Q}$ to arrive at the same result. By applying Eqs. (13) and (14)), the invariance under a Lorentz Boost transformation is easily seen. 


\section{SUMMARY}

We have shown how calculations in Special Relativity are facilitated by visualizing ticks of a clock as light-clock diamonds drawn on a sheet of rotated graph paper. When the relative-velocities between observers have rational Bondi-Doppler $k$-factors, the arithmetic and graphical constructions become very simple. This allows us to place emphasis first on the physical interpretation and geometrical modeling of situations in special relativity. Once established, one can then (if desired) advance to the development of the standard relativistic formulas.

\section{Acknowledgments}

I wish to thank my students in AST 110L and PHY 216 at Mount Holyoke College and in PHYS 63 at Bowdoin College, who tried out various worksheets based on this work. Their performance and feedback helped improve the presentation of the material. I also wish to thank Tevian Dray, Tom Moore, Stephen Naculich for useful comments.

\section{Appendix: Formulae for Velocity Composition and the Lorentz Boost Tranforma-} tion

For completeness, we show how one can obtain the formulae for Velocity Composition and the Lorentz Boost coordinate transformation using Radar Measurements. We follow the methods of Bondi 11 and Ellis. ${ }^{20}$ The starting point for both formulae begins with

$$
\begin{aligned}
t_{R}^{(\text {Alice })} & =k_{\text {Alice }, \text { Bob }} t_{S}^{(\text {Bob })} \\
t_{s}^{(B o b)} & =k_{\text {Alice }, \text { Bob }} t_{r}^{(\text {Alice })}
\end{aligned}
$$

from Fig. 16 and Eq. (15).

We first obtain the Velocity Composition formula, Eq. (20). From Sec. VG, $k_{\text {Alice }, \text { Carol }} / k_{\text {Bob,Carol }}=\sqrt{\left(t_{R}^{(\text {Alice })} / t_{S}^{(\text {Bob })}\right)\left(t_{s}^{(\text {Bob })} / t_{r}^{(\text {Alice })}\right)}=k_{\text {Alice }, \text { Bob }}$, since each term in paren-

thesis is equal to $k_{\text {Alice }, B o b}$. [Alternatively, write Eq. (A.1) as $t_{R}^{(\text {Alice })}=k_{\text {Alice }, \text { Bob }} t_{S}^{(\text {Bob })}=$ 
$k_{\text {Alice }, \text { Bob }}\left(k_{\text {Bob,Carol }} t_{W}^{(\text {Carol })}\right)=k_{\text {Alice,Carol }} t_{W}^{(\text {Carol })}$, using Fig. 16 and Eq. (15) again.] Thus,

$$
k_{\text {Alice }, \text { Carol }}=k_{\text {Alice }, \text { Bob }} k_{\text {Bob,Carol }},
$$

which embodies velocity composition. (Proof: Plug this expression into Eq. (28), then use Eq. (29) twice to obtain Eq. (20).)

We now obtain the Lorentz Boost coordinate transformation formulae, Eqs. (18) and (19). (It may help to recall Eqs. (21) and (22).) Using Eqs. (1A.1) and (A.2) with $k=k_{A l i c e, B o b}$, write

$$
\begin{aligned}
\frac{1}{2}\left(t_{R}^{(\text {Alice })} \pm t_{r}^{(\text {Alice })}\right) & =\frac{1}{2}\left(k t_{S}^{(\text {Bob })} \pm k^{-1} t_{s}^{(\text {Bob })}\right) \\
& =\frac{1}{2}\left(k\left(t_{W}^{(B o b)}+x_{W}^{(B o b)}\right) \pm k^{-1}\left(t_{W}^{(B o b)}-x_{W}^{(B o b)}\right)\right) \\
& =\left(\frac{k \pm k^{-1}}{2}\right) t_{W}^{(B o b)}+\left(\frac{k \mp k^{-1}}{2}\right) x_{W}^{(B o b)}
\end{aligned}
$$

where we used Eq. (22) to introduce Bob's rectangular coordinates of event $W$. Using Eq. (29), we have $\left(k+k^{-1}\right) / 2=1 / \sqrt{1-\beta^{2}}=\gamma$ and $\left(k-k^{-1}\right) / 2=\beta / \sqrt{1-\beta^{2}}=\beta \gamma$. With the upper signs in Eq. (A.4), the left-hand side is $t_{W}^{(\text {Alice) }}$ (using Eq.(21)), and we obtain the analogue of Eq. (18). With the lower signs, the left-hand side is $x_{W}^{(\text {Alice })}$, and we obtain the analogue of Eq. (19).

* Electronic address: rsalgado@bowdoin.edu

1 A. Einstein, "On the Electrodynamics of Moving Bodies" (1905) in The Principle of Relativity, by A. Einstein, H.A. Lorentz, H. Minkowski, and H. Weyl (Dover, New York, 1923), pp. 37-65.

2 H. Minkowski, "Space and Time" (1909) in The Principle of Relativity by H.A. Lorentz, A. Einstein, H. Minkowski, and H. Weyl, (Dover Publications, New York, 1923).

3 P.A.M. Dirac, "Forms of Relativistic Dynamics," Rev. Mod. Phys. 21, 392-399 (1949); L. Parker and G.M. Schmieg, "A useful form of the Minkowski diagram," Am. J. Phy. 38, 1298-1302 (1970). Note that some authors use alternate conventions for signs and factors of $1 / \sqrt{2}$, which would complicate the counting calculations.

4 S. Daubin, "A Geometrical Introduction to Special Relativity," Am. J. Phy. 30, 818-824 (1962); 
J. Aharoni, The Special Theory of Relativity (Oxford, Oxford, 1965), pp. 30-33; D. Bohm, The Special Theory of Relativity (Benjamin, New York, 1965), pp. 152-153; Y.S. Kim and M.E. Noz, "Dirac's light-cone coordinate system," Am. J. Phy. 50, 721-724 (1982).

5 N.D. Mermin, "Space-time intervals as light rectangles," Am. J. Phy. 66, 1077-1080 (1998).

6 R.B. Salgado, "Visualizing proper-time in Special Relativity," Physics Teacher (Indian Physical Society), 46, 132-143 (2004); available at arXiv:physics/0505134 v1 [physics.ed-ph].

7 D. Brill and T. Jacobson, "Spacetime and Euclidean geometry," General Relativity and Gravitation 38, 643-651 (2006).

8 G.W. Gibbons and S.N. Solodukhin, "The geometry of small causal diamonds," Phys. Lett. B, 649, 317-324 (2007).

9 The magnitude of the ordinary Euclidean area is equal to the magnitude of the area in Minkowski spacetime, up to a constant scalar factor. See Sec. VI and Ref. 7 .

10 We use units where temporal displacements are measured in units of "ticks" and spatial displacements are also measured in ticks, the times needed by light to travel those spatial displacements. In these units, velocities are dimensionless, and the speed of light is equal to one. In addition, we use the timelike-signature convention so that the square-interval is $d s^{2}=d t^{2}-d x^{2}$. We follow the usual convention where time runs upwards in the diagram.

11 H. Bondi, Relativity and Common Sense (Dover, New York, 1962).

12 A.A. Robb, Optical Geometry of Motion: A New View of the Theory of Relativity (Heffer, Cambridge, 1911).

13 R.F. Marzke and J.A. Wheeler, "Gravitation as Geometry-I: The Geometry of Space-Time and the Geometrodynamical Standard Meter," in Gravitation and Relativity, edited by H.-Y. Chui and W.F. Hoffman (Benjamin, New York, 1964), pp. 40-64; C.W. Misner, K.S. Thorne, and J.A. Wheeler, Gravitation (W.H. Freeman, New York, 1973);

14 J.L. Anderson and R. Gautreau, "Operational Approach to Space and Time Measurements in Flat Space," Am. J. Phy., 37, 178-189 (1969).

15 J.L. Synge, Relativity: The General Theory (North-Holland, Amsterdam, 1960).

16 E.F. Taylor and J.A. Wheeler, Spacetime Physics (W.H. Freeman, New York, 1966).

17 E.F. Taylor and J.A. Wheeler, Spacetime Physics (W.H. Freeman, New York, 1992), 2nd ed.

18 T.A. Moore, Six Ideas That Shaped Physics, unit R (McGraw-Hill, New York, 2002) 2nd ed.

19 R.P. Geroch, General Relativity from A to B (University of Chicago Press, Chicago, 1978). 
20 G.F.R. Ellis and R.M. Williams, Flat and Curved Space-times (Oxford, New York, 1988).

21 N.M.J. Woodhouse, Special Relativity (Springer-Verlag, London, 2003).

22 T. Dray, The Geometry of Special Relativity, 〈physics.oregonstate.edu/coursewikis/GSR/〉 (2011).

23 This is equivalent to Minkowski's definition: the radius vector drawn to a point on the hyperbola is perpendicular to the tangent vector at that point. (See Ref. 2, p. 85.)

24 In Minkowski spacetime, we have the dot-products: $\overrightarrow{O T} \cdot \overrightarrow{O T}=1, \overrightarrow{O X} \cdot \overrightarrow{O X}=-1$, and $\overrightarrow{O X} \cdot \overrightarrow{O T}=$ 0. Since $\overrightarrow{O M}=\frac{1}{2}(\overrightarrow{O T}-\overrightarrow{O X})$ and $\overrightarrow{O N}=\frac{1}{2}(\overrightarrow{O T}+\overrightarrow{O X})$, it follows that $\overrightarrow{O M} \cdot \overrightarrow{O N}=\frac{1}{2} \neq 0$.

25 This notion of "area" will be more fully explained in Sec. VI.

26 N. Calder, "Einstein's Universe", produced by the BBC, 1979. (Distributed by Corinth Films, 3117 Bursonville Rd., Riegelsville, PA 18077, 〈www.store.corinthfilms.com〉.)

27 We assume that Alice and Bob can suitably encode and decode the transmissions.

28 Indeed, this is one of the properties that makes Minkowski spacetime diagrams difficult to interpret. Note, however, that Galilean spacetime diagrams [ordinary position-time graphs] also have this property.

29 Since $(\Delta x)^{2}+(\Delta s)^{2}=(\Delta t)^{2}$ for nonnegative integers $\Delta x, \Delta s$, and $\Delta t$, with $\Delta x<\Delta t$ and $\Delta s \leq \Delta t$ for future-timelike displacements, then $(\Delta x, \Delta s, \Delta t)$ form a Pythagorean triple. When such triples are generated by $\Delta x=\lambda\left(m^{2}-n^{2}\right), \Delta s=\lambda(2 m n)$, and $\Delta t=\lambda\left(m^{2}+n^{2}\right)$, with positive integers $\lambda, m$, and $n$ (with $m \geq n$ ), we find that $k=m / n$. Alternatively, when the generator-formulas for $\Delta x$ and $\Delta s$ are switched (i.e., $\Delta s=\lambda\left(m^{2}-n^{2}\right)$ and $\Delta x=\lambda(2 m n)$ ), we find that $k=(m+n) /(m-n)$.

30 Based on Fig 7.6 (p. 207) in Taylor and Wheeler (Ref. 17). Note that the area of the massdiamond or the tickmarks of mass-units can now replace the "handles" on the momentumvectors, first described on p. 198. See also problem R10.S3 (p. 189) in Moore (Ref. 18). 